\title{
Detection and Characterization of Human Serum Antibodies to Polycyclic Aromatic Hydrocarbon Diol-epoxide DNA Adducts
}

\author{
Mark J. Newman, ${ }^{*}$ Bret A. Light, ${ }^{\ddagger}$ Ainsley Weston, ${ }^{\ddagger}$ David Tollurud, ${ }^{5}$ Jeff L. Clark, ${ }^{5}$ Dean L. Mann, ${ }^{\ddagger}$ \\ Joan P. Blackmon; ${ }^{\star}$ and Curtis C. Harris ${ }^{\ddagger}$ \\ *Veterinary Microbiology and Parasitology, School of Veterinary Medicine, Louisiana State University, Baton Rouge, \\ Louisiana 70803; ${ }^{\ddagger}$ Laboratory of Human Carcinogenesis and ${ }^{\S}$ Environmental Epidemiology Branch, \\ National Cancer Institute, National Institutes of Health, Bethesda, Maryland 20892
}

\begin{abstract}
The presence of serum antibodies to the diol-epoxide DNA adducts of representative polycyclic aromatic hydrocarbons (PAH), chrysene, benz[a]anthracene and benzo[a]pyrene, was determined by ELISA using serum samples obtained from normal healthy individuals. Antibodies that reacted against PAH adducted-DNA, but not against PAH-adducted protein, were found in the serum of $\sim 40 \%$ of the test individuals. Specificity analysis of the antibodies demonstrated that serological cross-reactions between the benzo[a]pyrene and the chrysene diol-epoxide adducts were present. Similar cross-reactivity between the benz[a]anthracene and the chrysene adducts was observed. Sera containing antibodies that were apparently specific for each of the three PAH-DNA adducts were also identified. The presence of antibodies to PAH-DNA adducts indicates both past exposure to these carcinogenic PAH and their metabolic activation to the DNA damaging metabolites. These antibodies may prove to be useful in both retrospective and prospective epidemiological studies of various diseases associated with PAH exposure.
\end{abstract}

\section{Introduction}

Polycyclic aromatic hydrocarbons (PAH) ${ }^{1}$ are a group of compounds that are present in the environment as the result of the combustion of organic matter. The sources and the levels of environmental contamination for many of these compounds have been determined (1). Human exposure to complex mixtures of PAH occurs primarily through three routes: $(i)$ the respiratory tract through the smoking of tobacco products $(2$, 3 ) and the inhalation of polluted air $(5,6)$, (ii) the gastrointesti-

Presented at the 71 st Annual Meeting of the Federation of American Societies for Experimental Biology, Washington, DC, 29 March-2 April 1987.

Address reprint requests to Dr. C. C. Harris, Building 37, Room 2C07, National Institutes of Health, Bethesda, MD 20892.

Received for publication 11 February 1987 and in revised form 26 January 1988.

1. Abbreviations used in this paper: BA, benz[a]anthracene; BADE, benz[a]anthracene-diol-epoxide; r-8,t-9-dihydroxy-t-10,11-oxy8,9,10,11-tetrahydrobenz[a]anthracene; BPDE, benzo[a]pyrene-diolepoxide; r-7, t-8-dehydroxy-t-9,10-oxy-7,8,9,10-tetrahydorbenzo[a]pyrene; BP, benzo[a]pyrene; ChDE, chrysene-diol-epoxide; r-7,t-2-dihydroxy-t-3,4-oxy-1,2,3,4-tetrahydrochrysene; NGS, normal goat serum; PAH, polycyclic aromatic hydrocarbons; SSC, $3 \mathrm{M} \mathrm{NaCl}+0.3$ $\mathrm{M}$ sodium citrate, $\mathrm{pH}$ 7.0.

The Journal of Clinical Investigation, Inc.

Volume 82, July 1988, 145-153 nal tract through the ingestion of contaminated drinking water (4) and broiled, smoked, or otherwise processed foods (7), and (iii) skin contact, which usually occurs from occupational exposure (8).

Many PAH have been recognized as common environmental pollutants and as potent carcinogens; benzo[a]pyrene (BP) has been studied most extensively and is considered as the prototype compound. BP can be activated metabolically to the ultimate-carcinogen, $7 \beta, 8 \alpha$-dihydroxy-9 $\alpha 10 \alpha$-epoxy-7, 8, 9, 10-tetrahydrobenzo[a]pyrene (BPDE). The activation of BP occurs through a multistep process that includes: $(i)$ oxidation across aromatic double bonds by the action of the mammalian cytochrome P450 enzyme system that results in the formation of simple epoxides, (ii) the formation of dihydrodiols by the action of epoxide hydrolases, and (iii) a second round of cytochrome P450 mediated oxidation at the site of olefinic double bonds to form the reactive diol-epoxides. The activated diol-epoxide binds primarily to the exocyclic amino groups of deoxyguanine in a covalent manner to form DNA adducts (9-11). The BPDE-DNA form is the major adduct that is detected in human tissues following experimental B[a]P exposure in vitro $(12,13)$ and in the DNA of peripheral blood lymphocytes $(14,15)$ and lung tissues (16) obtained from persons exposed environmentally to high levels of BP. The observations made concerning the metabolic activation and the formation of adducts using $\mathrm{B}[\mathrm{a}] \mathrm{P}$ are considered to be representative for PAH as a class of chemical carcinogens (17).

We reported recently that the occupational exposure of coke oven workers to BP led not only to the formation of BPDE-DNA adducts, as was expected, but also to the production of antibodies that reacted with an epitope(s) on BPDEDNA adducts (15). The levels of BP to which coke oven workers are exposed are known to be very high, much greater than those found in the normal environment (6), and as such the presence of BPDE-DNA adducts and/or antibodies to these adducts most likely reflects exposure to BP.

The detection of specific antibodies to adducts may serve as an indicator of environmental exposure of humans to BP as well as other common PAH. To test this hypothesis and to characterize further the immune response against PAH adducts we assayed the sera from 99 healthy individuals who were exposed to average urban environmental levels of PAH. The purpose of this study was to determine if persons from urban environments with typical PAH éxposure profiles responded immunologically to PAH-DNA adducts in a manner similar to that of the occupationally exposed coke oven workers. Data obtained from the characterization of this population can then be used as a baseline for the development of both retrospective and prospective environmental epidemiological studies involving PAH exposure of humans. The results of these studies are provided in this report. 


\section{Methods}

Human subjects; epidemiology and blood sample collection. Samples for this study were selected from a bank of several hundred frozen serum samples collected during a population-based survey of the Washington, DC metropolitan area. Briefly, the population selection process involved random digit dialing to select a stratified random sample of subjects. Demographic, lifestyle, and medical information was collected through telephone and self-administered questionnaires. Approximately one-third of potential study subjects were excluded on the basis of lifestyle characteristics (homosexual activity, intravenous drug use) or medical conditions (recent hospitalization, severe allergies, steroid medications, blood product transfusion since 1975 , connective tissue disease, or recent pregnancy) which might affect their immunologic competence. Phlebotomy was performed by a nurse phlebotomist at a specially equipped mobile van. Serum samples were collected in evacuated serum separator tubes, centrifuged $(1,200 \mathrm{~g}, 20$ $\mathrm{min}$ ) and refrigerated overnight. The following morning, serum was decanted and frozen in $1-\mathrm{ml}$ aliquots and stored at $-120^{\circ} \mathrm{C}$ until subsequent analysis.

100 samples for the current study were selected to represent approximately equal numbers of men and women including equal numbers of smokers and nonsmokers. Volunteers were divided among four age decades (ages 40-79). The sera were chosen without regard for occupational exposures, socioeconomic status or the results of other laboratory tests. One sample could not be analyzed for technical reasons. The remaining 99 samples were from 48 men and 51 women. Actual age range $40-76 \mathrm{yr}$ ). 49 of the subjects currently smoked cigarettes. Of the 50 nonsmokers, 22 had never regularly smoked cigarettes and 28 had smoked regularly at some time in the past. Complete epidemiologic data were available for 98 of the samples. Most study subjects (79\%) had completed high school and $62 \%$ reported some form of higher education. Approximately one-third of subjects were employed in professional or managerial positions and one-third in saleswork or clerical jobs with the remainder employed in skilled or semiskilled labor $(12 \%)$, technical or product sales $(9 \%)$, or unskilled labor $(7 \%)$. It should be noted that there are no major industrial or manufacturing sources of material known to contain substantial amounts of PAH (i.e., coal processing, asphalt production) in the greater Washington, DC metropolitan area. We have therefore considered this population to be typical in terms of exposure to PAH through urban environmental pollution, such as automobile and diesel exhaust.

Synthetic PAH-DNA adduct preparation. Small quantities (1 mg) of synthetic vicinal diol-epoxides of BPDE, benz[a]anthracene (BADE), and chrysene (ChDE) were obtained from the NCI Carcinogen Reference Standard Repository (Bethesda, MD). These compounds were dissolved in ethanol:tetrahydrofuran (24:1) and reacted in the dark at room temperature with calf thymus DNA $(10 \mathrm{mg}$; Sigma Chemical Co., St. Louis, MO) in aqueous solution $(1 \mathrm{mg} / \mathrm{ml})$. After $16 \mathrm{~h}$ the reaction mixture was extracted eight times with diethyl-ether and the DNA was precipitated from the aqueous layer by the addition of sodium chloride (100 mM, final concentration) and ethanol (2.5 vol). The samples of PAH-DNA were washed in $70 \%$ ethanol and redissolved in distilled water. The level of modification for the BPDE-DNA was determined both by liquid scintillation counting (because the BPDE was ${ }^{3} \mathrm{H}$-labeled) and by measuring the absorbance spectrum of the sample $\left(\triangle \mathrm{E} 29 \times 10^{3}\right.$ at $\left.347 \mathrm{~nm}\right)(18)$. The outer two diol-epoxides that were used for these modifications were not radiolabeled and estimates of the levels of modification were made by measuring the absorbance spectra of the modified DNA samples; epsilon values for each of the chromophores studied are given in the spectral atlas of polyacyclic aromatic compounds (19). Additional confirmatory evidence for the levels of modification that were obtained in each case were determined by analytical ${ }^{32} \mathrm{P}$-postlabeling (20). The level of modification of DNA by the BPDE was determined to be $1 \%$; the levels of DNA modification achieved using the BADE and the ChDE were between 0.1 and $0.2 \%$. The fluorescence excitation, emission and synchronous spectra were found to be identical to those of phenanthrene for both the BADE and the ChDE-DNA adducts, the fluorescence spectral properties of the BPDE-DNA adducts were pyrene-like.

These PAH were chosen as representative because the parent hydrocarbons are all present in comparable concentrations in the complex mixtures of PAH found in the environment (1) and then in turn are metabolized to their respective diol-epoxides that form adducts with DNA and probably with cellular proteins such as hemoglobin. The potent carcinogenic properties of BP have been established in experimental animals. Chrysene and BA have been found to be only weakly carcinogenic in the biologic systems that have been tested thus far (21). The chemical structures of the adducts used in this study are shown in Fig. 1.

Synthetic BPDE-globin preparation. Human globin (Sigma Chemical Co.) was adducted with $\left[{ }^{3} \mathrm{H}\right] \mathrm{BPDE}$ (NCI Carcinogen Reference Standard Repository) using the same basic methods as for the production of PAH-DNA adducts. The $\left[{ }^{3} \mathrm{H}\right] \mathrm{BPDE}$ was dissolved in the ethanol/tetrahydrofuran solution at a concentration of $1 \mathrm{mg} / \mathrm{ml}$ and mixed with $2 \mathrm{ml}$ of the globin solution $(50 \mathrm{mg} / \mathrm{ml}$ in PBS). Adducted globin was precipitated and recovered by multiple extractions with organic solvents as described for the preparation of PAH-DNA adducts. The precipitated globin was dissolved in PBS (22). The amount of $\left[{ }^{3} \mathrm{H}\right] \mathrm{BPDE}$ adducted to the globin was determined to be $16.9 \mathrm{pM}$ $\mathrm{BPDE} / \mu \mathrm{g}$ of globin by beta scintilation counting. This is a modification level of $0.4-0.6 \%$ by weight, which was only slightly less than that which was achieved in the formation of DNA-adducts. Similar globin adducts could not be produced using the BADE or the ChDE because these compounds are not currently available in radioactively labeled forms and as such, modification levels could not have been determined readily.

Detection of antibodies to PAH-DNA adducts; direct antibody binding ELISA. The PAH-modified and nonmodified DNA were used as the standard test antigens. These were attached to polyvinylchloride microtiter plates (Costar, Cambridge, MA) by drying at $37^{\circ} \mathrm{C}$ for $12 \mathrm{~h}$ at the concentration of $20 \mathrm{ng} /$ well in $50 \mu$ l of $20 \times$ SSC buffer ( $3 \mathrm{M}$ $\mathrm{NaCl}, 0.3 \mathrm{M}$ sodium citrate, $\mathrm{pH} 7.0$ ). We have used the ELISA described in the subsequent paragraph, and anti-DNA monoclonal antibodies from our laboratory to demonstrate that control DNA (nonmodified) and PAH modified DNA bind to the wells of the test plate in equal quantities. Wells coated with the nonmodified DNA or without DNA (SSC buffer alone) were used as controls. The wells without DNA were used to establish background binding levels of the test sera and the

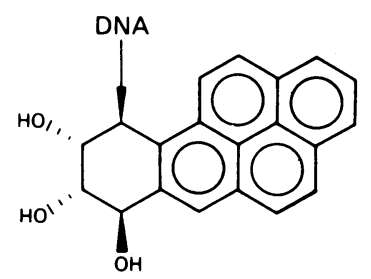

Benzola)pyrene - DNA adduct

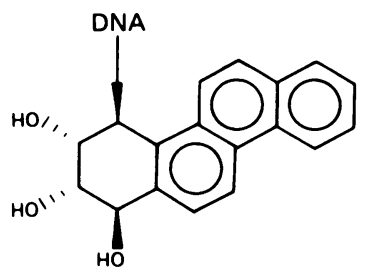

Chrysene - DNA adduct

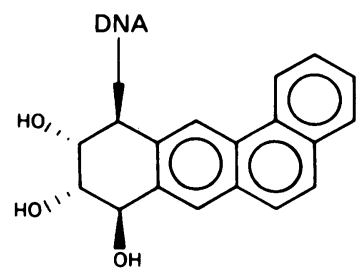

Benzla)anthracene - DNA adduct
Figure 1. Chemical structures of the diol-epoxide forms of the three PAH used; B[a]P, B[a]A, and chrysene. 
wells coated with nonmodified DNA were used to distinguish antibodies that bind to DNA from those that bind to PAH-DNA (15).

DNA-coated test plates were prepared for the assay by multiple washing steps using distilled water to remove salt crystals followed by a 30-min room temperature incubation of all wells with $150 \mu \mathrm{l}$ of PBS containing $10 \%$ (vol/vol) normal goat serum (PBS-NGS), to block protein binding sites on the polyvinylchloride solid phase. Human sera were tested in triplicate using 3 or $4 \log _{5}$ serial dilutions, with all dilutions being made in the PBS-NGS solution. Test sera ( $50 \mu \mathrm{l} /$ well) were incubated for $60 \mathrm{~min}$ with the antigen containing wells and then washed five times with PBS. The binding of human immunoglobulin was then detected using the biotinylated goat anti-human immunoglobulin reagents and the avidin-biotin horseradish-peroxidase system (ABC Vectastain, Vector Laboratories, Burlingame, CA) as described by the manufacturer. The enzyme reaction was developed by the addition of $100 \mu \mathrm{l}$ of the substrate solution $(0.05 \mathrm{M}$ citrate buffer, $\mathrm{pH} \mathrm{4.0;1}$ $\mathrm{mg} / \mathrm{ml}$ ortho-phenylenediamine plus $0.5 \mu \mathrm{l} / \mathrm{ml}$ of $30 \% \mathrm{H}_{2} \mathrm{O}_{2}$ to each of the wells. The enzymatic reaction was stopped after a 20-min room temperature incubation by the addition of $50 \mu \mathrm{l}$ of $2.0 \mathrm{M} \mathrm{H}_{2} \mathrm{SO}_{4}$. Plates were read using an automatic ELISA reader (Dynatech Laboratories, Alexandria, VA) and the absorbance at $490 \mathrm{~nm}$ was recorded. An anti-human immunoglobulin reagent that reacted with all isotypes of serum immunoglobulins was used. Each ELISA included the testing of a serum sample on modified and nonmodified DNA as well as on wells without any type of bound PAH-DNA (15).

Antibody reactions to each of the DNA-adducts were scored as positive when the mean absorbance value of triplicate test wells was significantly greater than that observed in triplicate control wells containing nonmodified-DNA as the test antigen. Significant ELISA absorbance levels were established by adding two standard deviations to the control absorbance values. Antibody titers were determined as the highest dilution of serum for which significant ELISA absorbance levels were obtained.

Antigen-competition ELISA. Selected serum samples that were found to contain antibodies that reacted against the PAH-DNA adducts were tested further using a competitive ELISA. In this assay the binding of antibodies to the solid-phase antigen was competed by the preincubation of the test serum with different test antigens before testing in the direct binding ELISA. Serum samples were diluted to a point at which binding was $80-90 \%$ maximal. The diluted serum samples were then mixed with varying amounts of modified or nonmodified DNA $(400,200$, and $100 \mathrm{ng} / \mathrm{ml})$ and incubated at room temperature for $60 \mathrm{~min}$. These serum/PAH-DNA mixtures were then tested as described for the direct binding ELISA against both the modified and nonmodified DNA. The results are expressed as "percent inhibition"; which was determined by using the levels of antibody binding of each diluted serum sample without any competing antigens as the level of maximal binding and the background antibody binding of each sample as the level of minimal antibody binding (15). The formula for determining this is as follows: (MAX - BKGD) - (TEST - BKGD)/(MAX - BKGD) $\times 100$, where MAX, the ELISA absorbance value of triplicate test wells which was observed using the monoclonal antibodies without any competing antigens; TEST, the ELISA absorbance value of triplicate test wells which was observed using monoclonal antibodies after incubation with competing antigens; BKGD, the ELISA absorbance value of triplicate test wells which was observed using no antigens on the solid phase, a measure of the background binding of the monoclonal antibodies to the polyvinylchloride surface of the ELISA test plates.

Competition binding was done using BPDE-, BADE-, ChDEDNA, and control-DNA as both competing antigens and as solidphase test antigens in all combinations.

Competition binding was also assayed using soluble, nonmetabolized BP, BA and chrysene as the competing antigens. Crystalized BP, BA and chrysene (Aldrich Chemical Co., Milwaukee, WI) were dissolved in dimethyl sulfoxide (Sigma Chemical $\mathrm{Co}$.) and then diluted in PBS to a concentration of $1 \mathrm{mg} / \mathrm{ml}$ PAH plus $10 \%$ (vol/vol) dimethyl sulfoxide in PBS and stored at $-65^{\circ} \mathrm{C}$. This solution was diluted fur- ther as required using the $10 \%$ dimethyl sulfoxide-PBS solution. The antigen competition assays were run as described for the PAH-DNA adducts using the soluble PAH at concentrations of 400,200 , and 100 $\mathrm{ng} / \mathrm{ml}$.

ELISA using BPDE-globin. The BPDE-globin and control-globin were attached to the wells of polyvinylchloride test plates by an overnight room temperature incubation of $200 \mathrm{ng} /$ well total protein in 25 $\mu \mathrm{l}$ of $0.06 \mathrm{M}$ bicarbonate buffer, $\mathrm{pH}$ 9.6. The direct binding ELISA using globin was run exactly as described for the PAH-DNA adducts. The antigen competition ELISA was also run as described for the PAH-DNA adducts using BPDE- and control-globin at concentration of 4,2 , and $1 \mu \mathrm{g} / \mathrm{ml}$ as the competing antigens in the fluid phase.

The reasons for running the two forms of the ELISA, direct antibody binding and antigen competition, are as follows: $(a)$ the results from the different forms of the ELISA were interpreted together to determine which PAH-DNA preparations share serologically defined epitopes; $(b)$ the results from the different forms of the ELISA allowed us to compare the DNA and globin adducts to determine the immunological relatedness of these compounds; $(c)$ the results from the different forms of the ELISA allowed us to identify those antibodies which recognized epitopes on PAH-DNA adduct preparations in the fluid phase and in the solid phase; an important consideration since attachment of antigens to the polyvinylchloride solid phase may distort the normal tertiary structure of the PAH-DNA adducts; $(d)$ the results of the different forms of the ELISA allowed us the opportunity to partially quantitate the antibody binding with respect to the preference of serum antibodies for a particular PAH-DNA adduct.

Statistical analysis. Basic statistical analysis were done using the SAS program package (SAS Institute, Inc., Cary, NC); specific programs are identified as they are referenced in the next section. Epidemiologic organization of this study and subsequent analysis were done as described $(23,24)$.

\section{Results}

Serological testing was done initially using the direct binding ELISA and DNA modified by adduction with the diol-epoxide forms of three representative PAH, BP, BA and chrysene, as the test antigens. Of the 99 sera tested, 43 had antibody binding patterns that demonstrated the presence of adduct specific antibodies. Representative adduct specific antibody binding profiles are shown in Fig. 2.

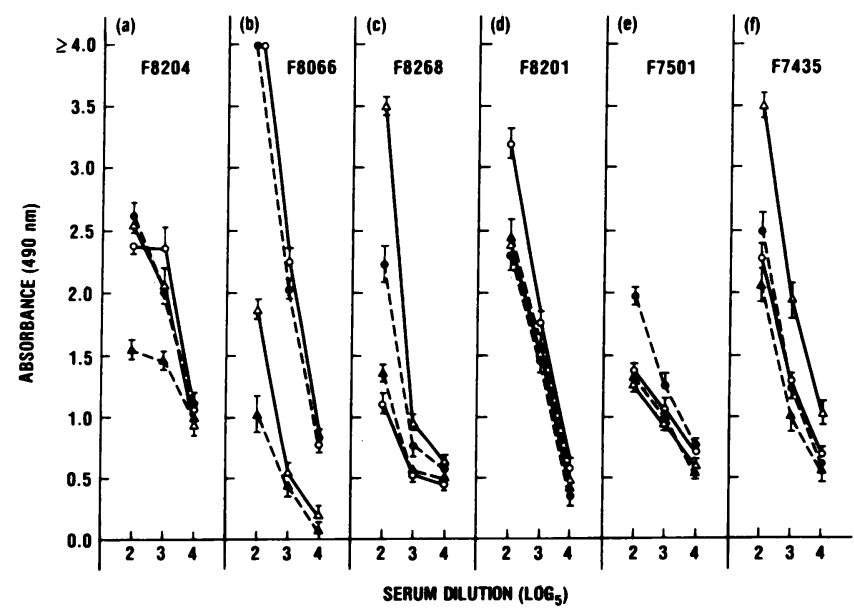

Figure 2. Antibody binding curves of human sera against BPDE-, BADE-, ChDE-, and control-DNA as determined by ELISA. The data values shown represent the mean $(\overline{\mathbf{x}})$ of triplicate tests \pm 1 SD. Antibody binding to each of the different adducts is as follows: $(0)$

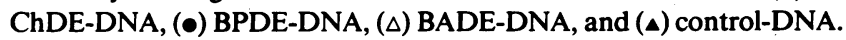


The presence of antibodies in a single serum that reacted against the three different PAH-DNA adducts was a common feature. Sera from 27 individuals reacted against all three types of the PAH-DNA adducts at one or more of the serum dilutions tested. These complex sera exhibited antibody binding curves similar to that shown for serum F8204 (Fig. $2 A$ ), in which the three different adduct preparations were recognized similarly, based on ELISA absorbance values and serum titrations. Four sera reacted in a preferential manner against two forms of the PAH-DNA adducts producing antibody binding patterns similar to those shown for serum F8066 (Fig. 2 B) with higher levels of antibody binding for BPDE-DNA and ChDE-DNA than for BADE-DNA, and serum F8268 which had antibodies only to BPDE-DNA and BADE-DNA (Fig. 2 $C)$. 12 sera reacted significantly against only a single PAHDNA adduct as shown for the sera F8201, F7501, and F7435, which bound to ChDE-DNA, BPDE-DNA, and BADE-DNA, respectively (Fig. 2, D-F).

The presence of antibodies to more than a single adduct in individual sera appeared to be more common than the presence of antibodies to only a single adduct. We analyzed these serological data using a $2 \times 2 \times 2$ chi-squared contigency table with four degrees of freedom, using the CATMOD program (SAS Institute, Inc.); serological reactions were scored simply as either positive or negative with respect to each adduct. The distribution of the serological reactions against the three test adducts is shown in Fig. 3 along with the numbers of positive reactions expected assuming that antibody reactions were independent of each other. This analysis demonstrates that sera with antibodies against all three adduct types occurred much more frequently than was expected. Similarly, sera which were totally negative, in terms of antibodies to all of the adducts used, were identified much more frequently. This distribution of the data is significantly different than would be expected for independent antibody reactions against each of the adducts $(P$ $<0.001$ ).

The identification of sera with antibody reactivity against a single type of PAH-DNA adduct was a rare occurrence, how-

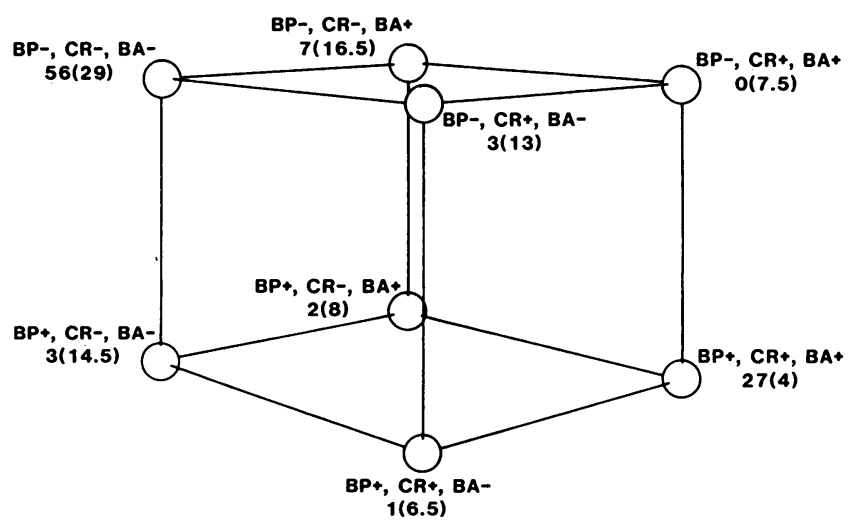

Figure 3. Observed distribution of antibody reactions for 99 sera against the three test adducts (given as BP, BA, and CR for BPDEDNA, BADE-DNA, and ChDE-DNA, respectively). The percentages of the total-panel identified with each adduct combination specificity are given in numerical fashion; the values in the parentheses represent the percentages of expected sera with each particular adduct combination specificity assuming that the presence of antibodies in all sera to each of the adducts is an independent event. ever, their identification suggested that each of these adducts may possess at least one immunologically unique epitope. The presence of antibodies to more than a single type of PAHDNA adduct in a serum was common and is also of interest in immunological terms. These complex binding patterns could be caused by the presence within a single serum of several specific antibodies against each of the different PAH-DNA adducts or these reactions may be due to the binding of antibodies that recognized a shared or cross-reactive epitope present on more than one of the chemical forms of PAH-DNA adducts. The antibody specificities of selected sera were therefore defined more rigorously using an antigen competition ELISA, an assay in which sera were preincubated with the different PAH-DNA adducts before being tested by ELISA to determine if antibodies with certain specificities can be inhibited. The results of these assays demonstrated that many of the complex sera did contain antibodies that were cross-reactive (Fig. 4). Analysis of one of these sera, F8066, demonstrated that BPDE-DNA was capable of inhibiting the binding of serum antibodies to both BPDE-DNA (Fig. $4 A$ ) and ChDEDNA (Fig. $4 B$ ). The reverse situation was not true, ChDEDNA did not inhibit the antibody binding to BPDE-DNA (Fig. $4 \mathrm{~A}$ ). However, BADE-DNA was also used successfully to partially inhibit antibodies from binding to ChDE-DNA (Fig. $4 B$ ) thus documenting some degree of serological between these two different adducts. Cross-reactivity between BPDEDNA and BADE-DNA could not be similarly demonstrated by antigen competition ELISA (Fig. $4, A$ and $C$ ). Similar results were obtained using five other complex sera (data not shown). These data suggested that two different cross-reactive epitopes are present on the different PAH-DNA adducts used in this study, one on BPDE-DNA and one on BADE-DNA, each of which is present on the ChDE-DNA.

The ability of ChDE-DNA and BPDE-DNA to inhibit an-

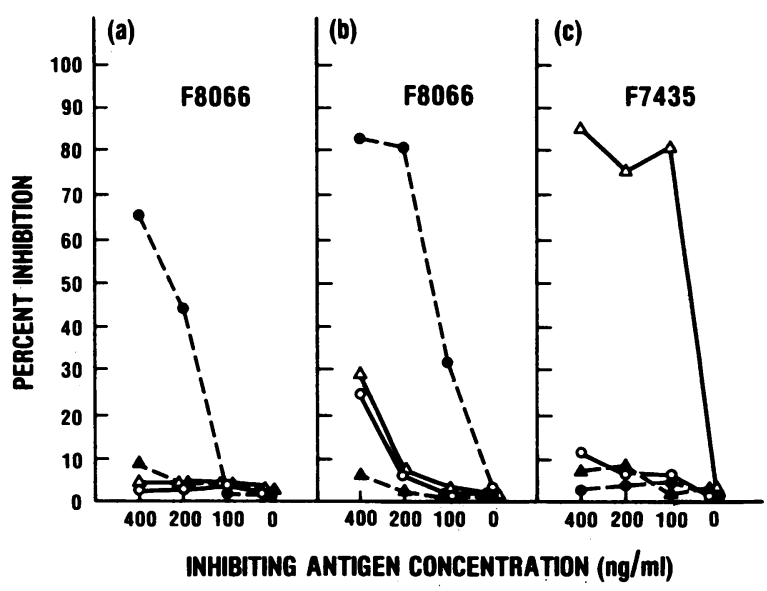

Figure 4. Antigen competition curves obtained using human sera containing antibodies to PAH-DNA adducts as determined by ELISA. The results are expressed as "percent inhibition" of binding which was determined using the binding of each serum without any competing antigen as the maximum binding level and the background binding of each serum as the level of minimum antibody binding level. The binding of the serum F8066 to BPDE-DNA following preincubation with BPDE-DNA (๑), ChDE-DNA (0), BADEDNA $(\Delta)$, and control-DNA $(\triangle)$ is shown in a. The binding patterns of serum samples F8066 to ChDE-DNA and F7435 to BADE-DNA following the same treatments are shown in $b$ and $c$, respectively. 
tibodies in serum F8066 from binding to BADE-DNA could not be established accurately. The antibodies to BADE-DNA in this serum appeared to represent only a minor portion of the total PAH-DNA specific antibodies present and our inability to assess their function in the antigen competition ELISA was probably limited by this feature. However, serum F7435, which reacted with only a single chemical form of the PAHDNA adducts (BADE-DNA specific) (Fig. $2 F$ ), was inhibited by only BADE-DNA (Fig. $4 C$ ); BPDE-DNA and ChDE-DNA failed to compete for antibody binding. The analysis of this serum demonstrates the BADE-DNA can serve as an antigen and that it possesses an immunologicaly unique epitope. Similar analyses were done with the other sera that reacted against only a single adduct. The titers of many of these were to low to be informative however, inhibition of two BPDE-DNA specific sera was accomplished with only BPDE-DNA, inhibition of antibody binding by ChDE-DNA and BADE-DNA was not observed (data not shown). These data demonstrate that BPDE-DNA also possesses an epitope unique to this adduct, similar to the data for BADE-DNA serum F7435 (Fig. $4 C$ ). Similar data for ChDE-DNA was not obtained inspite of the apparent presence of ChDE-DNA specific sera, such as F8201 (Fig. $2 \mathrm{D}$ ). This probably represents a combination of a lack of sensitivity in our ELISA system and low titered ChDE-DNA specific sera.

Analysis of the direct antibody binding ELISA data in conjunction with the antigen competition ELISA data demonstrated both the presence of antibodies specific for each of the PAH-DNA adducts used, and therefore suggests the presence of epitopes unique to at least two of the PAH tested, and the presence of antibodies that cross-react with the different adducts, suggesting the presence of shared epitopes. These data also demonstrated agreement between the direct binding and the antigen competition forms of the ELISA thus alleviating our concerns that the attachment of PAH-DNA adduct preparations to the polyvinylchloride assay plates might alter the antigenic structure of the adducts. Antibodies that bound to PAH-DNA adducts were never inhibited by nonmodified DNA demonstrating that the specificities of the serum antibodies were produced by alterations in the DNA resulting from the presence of an adduct.

Selected sera were assayed further to characterize the specificities of the antibodies to PAH. Antigen competition ELISA using the parent hydrocarbons (B[a]P, B[a]A and chrysene) as the competing antigens established that antibodies which bound to the BPDE-DNA, BADE-DNA, or the ChDE-DNA adducts were not inhibited by these simple chemical moieties even at concentrations 100 times greater than those present as PAH-DNA adducts (data not shown). The possibility that the serum antibodies could bind to PAH conjugated to protein was investigated using BPDE-globin and nonmodified-globin as the test antigens. Antibody binding to the BPDE-globin was not detected using either the direct binding ELISA or the antigen competition ELISA (Fig. 5). These data demonstrated that the serum antibodies are specific for PAH-DNA adducts. We believe this to indicate that the PAH-DNA adducts were probably the immunogens against which the antibodies were produced, since serum antibody reactivity was not detected against PAH-protein complexes or free PAH.

The presence or absence of antibodies to each of the PAHDNA adducts was compared to the gender, age, and to the current and past smoking status of the individual serum
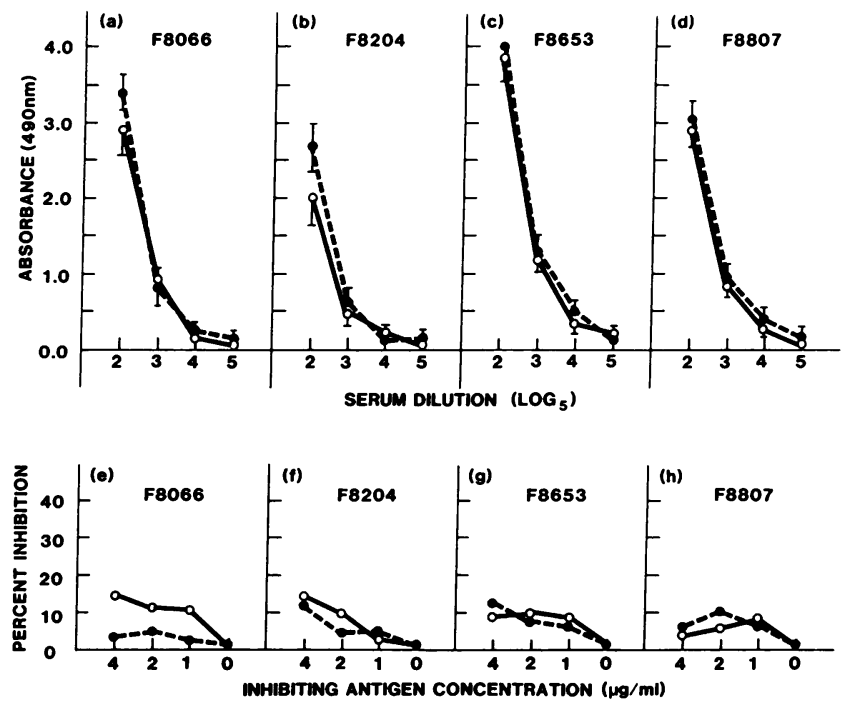

Figure 5. Antibody binding and antigen competition curves of four representative human sera against BPDE-globin and control-globin as determined by ELISA. Sera F8066 and F8204 were known to contain antibodies to BPDE-DNA (Fig. 2), sera F8653 and F8807 were characterized as BPDE-DNA antibody negative. The data values shown for the direct binding ELISA $(a-d)$ represent the mean $(\overline{\mathbf{x}})$ of triplicate tests $\pm 1 \mathrm{SD}$. Antibody binding to each of the test antigens is as follows: (๑) BPDE-globin, (o) control-globin. The antigen competition results are expressed as percent inhibition as described in the text. The binding of the four test sera to BPDE-DNA following preincubation with BPDE-globin (๑), and control-globin (O) is shown in $\mathrm{e}-\mathrm{h}$.

donors (Table I). Higher percentages of females produced antibodies against of all the PAH-DNA adducts in most of the different test groupings. The percentage of each of the female smoking status groups (current smoker, past smoker, or never regular smoker), which was positive for PAH-DNA antibodies, varied from 21 to $42 \%$ for the different groups; the lowest proportions of positive sera were obtained from past smokers (Table I). Most of the female smoking status groupings had $\sim 40 \%$ of their members classified as antibody positive. In the male volunteer group there was an inverse relationship between the smoking status and the presence of antibodies to PAH-DNA adducts. The highest percentages of antibody positive sera came from men that had never smoked regularly (Table I). The relevance of these data needs to be viewed cautiously at present due to the small sample size and because

Table I. Percentages of Antibody Positive Individuals within Test Groups Based on Gender and Smoking Status

\begin{tabular}{cccccccccc}
\hline & & \multicolumn{2}{c}{ Current smokers } & & \multicolumn{2}{c}{ Past smokers } & & \multicolumn{2}{c}{ Never smokers } \\
$\begin{array}{c}\text { PAH-DNA } \\
\text { adduct }\end{array}$ & $\begin{array}{c}\text { Total } \\
\text { sample }\end{array}$ & Male & Female & & Male & Female & & Male & Female \\
\hline & $n=99$ & $n=24$ & $n=25$ & & $n=14$ & $n=14$ & & $n=10$ & $n=12$ \\
BPDE & 33 & 25 & 44 & & 14 & 29 & & 30 & 42 \\
ChDE & 30 & 40 & 21 & & 21 & 30 & & 40 & 42 \\
BADE & 36 & 33 & 40 & & 14 & 36 & & 40 & 42
\end{tabular}

Data are presented as the percentage of antibody positive individuals within each smoking status grouping. Total test group size is given as $n$. 
Table II. Percentage of Antibody Positive Individuals within Test Groups Based on Gender and Total Lifetime Cigarette Consumption

\begin{tabular}{|c|c|c|c|c|c|c|c|c|}
\hline \multirow{2}{*}{$\begin{array}{l}\text { Pack years } \\
\text { estimate }^{*}\end{array}$} & \multicolumn{2}{|c|}{ Test group number } & \multicolumn{3}{|c|}{ Females $(n=51)^{\ddagger}$} & \multicolumn{3}{|c|}{ Males $(n=48)^{\ddagger}$} \\
\hline & Male & Female & BPDE & ChDE & BADE & BPDE & ChDE & BADE \\
\hline 0 & 10 & 12 & 42 & 42 & 42 & 30 & 40 & 40 \\
\hline $1-20$ & 8 & 19 & 47 & 47 & 42 & 0 & 0 & 0 \\
\hline $21-40$ & 13 & 6 & 17 & 0 & 33 & 23 & 15 & 31 \\
\hline $41-60$ & 12 & 7 & 43 & 29 & 43 & 25 & 25 & 42 \\
\hline$>60$ & 6 & 7 & 43 & 43 & 43 & 67 & 67 & 50 \\
\hline
\end{tabular}

* Pack years estimate, the equivalent of smoking one pack of cigarettes (20) per day per year. ${ }^{\ddagger}$ Results presented in the table are expressed as the percentage of antibody positive individuals within each group; test group size is given as $n$. Total test group size for this table is 98 ; one male individual was removed from the analysis because of incomplete epidemiological data concerning the estimation of pack years.

almost all of the persons (both females and males) contributing sera to these studies had smoked at least one time during their lives. Exposure through passive smoking was not controlled in this study either. We therefore cannot preclude the possibility that the primary antibody responses were produced against DNA adducts formed from PAH contained in tobacco smoke.

Analysis of the antibody data using total cigarette comsumption, as estimated by pack years, using a pooled sample of current and past smokers, revealed a difference in the responses that was associated with the gender of the volunteers. Females with antibodies in their sera to PAH-DNA adducts were readily identified in all of the test groupings, regardless of their total cigarette consumption. The highest proportions of antibody positive donors was in the group with the lowest total cigarette consumption levels, 1-20 pack years. Male serum donors with antibodies to any of the adducts were not found in this lowest pack years group (Table II). The percentage of antibody positive females remained similar as pack year estimates increased. However, the proportion of antibody positive males increased as pack years estimates increased.

We analyzed these data further by ranking the serum donors with positive smoking histories according to their pack years estimates and genders. These groups were then divided into terciles of similar sizes based on pack years estimates. The terciles distribution for the males was as follows: (a) 1-25 pack years, median of 13 pack years, 12 individuals; $(b) 26-45$ pack years, median of 36 pack years, 11 individuals; $(c) \geq 46$ pack years, median of 55 pack years, 15 individuals.

The tercile distribution for the females was as follows: $(a)$ 1-15 pack years, median of 10 pack years, 12 individuals; (b) 16-40 pack years, median of 22 pack years, 13 individuals; $(c)$ $\geq 41$ pack years, median of 60 pack years, 14 individuals.

The data were examined across terciles using the extended Mantel-Haenszel test. We found that the increases in the percentages of antibody positive males was associated with increases in pack years estimates but this trend did not quite reach a significant level $(P<0.072)$. There was no association between the pack years estimates and the presence of antibodies to PAH-DNA adducts for the female group. These data suggested a gender-exposure interaction in the male volunteers and a smoking status-exposure interaction as well since former smokers tended to have a lower cumulative exposure than the current smokers.

Antibody titers were estimated for all of the test sera as quantitative measurements of the PAH-DNA specific antibodies. The titers for antibodies to each of the three types of PAH-
DNA adducts are listed in Table III. In general, antibody titers were low with most values in the 25-125 range. There was no correlation of antibody titer with gender, smoking history, or the age of the volunteer.

\section{Discussion}

We have reported that the serum from coke oven workers frequently contain antibodies against PAH-DNA adducts, the testing was done using BPDE-DNA (15). These workers are exposed routinely to very high levels of PAH in their occupational environment and therefore, the presence of these antibodies is thought to reflect PAH exposure levels. The results of the present study demonstrate that persons with levels of exposure to PAH typical of urban environments also produce antibodies to BPDE-DNA as well as to the DNA adducts of the related PAH, BADE, and ChDE (Fig. 1). These data support our initial observation that persons are capable of producing an immune response against certain PAH-DNA adducts. These current data further our initial observation in that they demonstrate that typical environmental levels of PAH exposure can serve to induce an immune response and that antibodies are produced against more than a single type of PAHDNA adduct (Fig. 2).

The specificity of the serum antibodies for PAH-DNA adducts was readily demonstrated using the direct binding ELISA and the antigen competition ELISA. The results of these assays showed that the antibodies reacted against PAH adducts on DNA only; BPDE-globin and free BP, BA and

Table III. Antibody Titers of Test Serum Panel to the Three PAH-DNA Test Adducts

\begin{tabular}{ccccc}
\hline & \multicolumn{4}{c}{ Titer determinations* } \\
\cline { 2 - 5 } $\begin{array}{c}\text { PAH-DNA } \\
\text { adduct }\end{array}$ & $<25$ & 25 & 125 & 2625 \\
\hline BPDE & 67 & 19 & 10 & 4 \\
ChDE & 70 & 17 & 10 & 3 \\
BADE & 64 & 15 & 15 & 3
\end{tabular}

Results are as percentages of the total test serum panel of 99 .

* Titers were determined as described in the text as the largest dilution for which significant antibody binding could be detected. Log 5 dilutions were used; the titer determination values represent the reciprocal of the actual serum dilution. 
chrysene failed to compete with the PAH-DNA for antibody binding. This observation has lead us to propose that the PAH-DNA adducts were the antigens against which the antibody responses were produced. The presence of these antibodies must therefore reflect both exposure to the specific PAH and metabolic activation of the PAH to their electrophilic forms (9-13).

Antibodies specific for a particular PAH-DNA adduct and antibodies that recognized more than a single PAH-DNA adduct were also identified. Of particular interest were those sera with antibodies that bound to both ChDE-DNA and BPDEDNA. In the example shown (Fig. $4 A$ ), the binding of antibodies in serum F8066 to solid-phase BPDE-DNA could be inhibited by fluid-phase BPDE-DNA but not by fluid-phase ChDEDNA. This was in contrast to what was expected since high levels of antibody binding to ChDE-DNA could be observed using the direct antibody binding ELISA (Fig. $2 B$ ). Fluidphase BPDE-DNA could also inhibit the binding of serum F8066 antibodies to solid-phase ChDE-DNA, to a greater extent than fluid-phase ChDE-DNA could be used to inhibit antibody binding to solid-phase ChDE-DNA (Fig. $4 \mathrm{~B}$ ). The reverse situation was not true, fluid phase ChDE-DNA could not inhibit antibody binding to solid phase BPDE-DNA. These data clearly demonstrate an antibody binding preference in serum F8066 for the BPDE-DNA over the ChDEDNA; the major portion of total antibody binding was to BPDE-DNA when both of the PAH-DNA were present in the same ELISA test well. We have therefore considered the antibodies in this serum as being directed against BPDE-DNA and the binding to ChDE-DNA was considered to be a serological cross-reaction. Similar cross-reactivity between ChDE-DNA and BADE-DNA was observed using this serum (Fig. $4 \mathrm{~B}$ ). However, cross-reactivity between BADE-DNA and BPDEDNA was not observed using serum F8066 (Fig. $4 \mathrm{~A}$ ), serum F7435 (Fig. $4 C$ ), or five other sera with complex antibody binding profiles and several very specific sera (data not shown). These data have lead us to hypothesize that antibodies are more readily produced against the BPDE-DNA and BADE-DNA adducts and further that the binding against the ChDE-DNA adducts is due primarily to serological cross-reactivity.

The combination of direct antibody binding ELISA and antigen competition ELISA proved to be very valuable for determining antibody specificity, as described in the preceding paragraph. However, we felt it was also important to document the binding of human antibodies to PAH-DNA adducts using a fluid-phase assay system since the attachment of antigens to a solid phase may alter or destroy certain epitopes. Our data demonstrated that the antibodies to PAH-DNA adducts can bind to both fluid-phase and solid-phase BPDE-DNA and BADE-DNA. Both of these PAH-DNA adduct preparations were recognized and bound by antibodies in the fluid phase and thus they were able to inhibit the antibody binding to the solid-phase adducts by $68-88 \%$. In both cases we were not able to inhibit antibody binding to the solid-phase antigen totally by fluid-phase PAH adducted DNA when the antigens were present in both phases in the same quantities $(400 \mathrm{ng} / \mathrm{ml} ; 20$ $\mathrm{ng} / \mathrm{well}$ ). We believe this represents a condition of equilibrium binding of the antibodies whereby the antibodies can dissociate from either the fluid- or solid-phase antigen and then bind to the other antigen phase. Complete inhibition of antibody binding to solid phase PAH-DNA by fluid-phase adducts would therefore not be predicted as was the situation in this study. However, this was not the case when the ChDE-DNA was involved and BPDE-DNA were used as competing antigens since most of the test serum antibodies appeared to recognize BPDE-DNA preferrentially. Further evaluation of these observations will require that equilibrium binding experiments be done. These experiments represent the topic of future studies.

Although antibodies to PAH-DNA adducts were produced at detectable levels by many of the volunteers in the test population (43\%), this did not represent the majority of our test group. The reasons for this are unknown but may be associated with different levels of PAH-exposure, a variable that could not be accurately assessed in this study. Data obtained previously (15) using coke oven workers as serum donors were very similar with respect to $\mathrm{B}[\mathrm{a}] \mathrm{P}$; only $28 \%$ of the persons sampled had antibodies to BPDE-DNA compared to $33 \%$ found in this study. Coke oven workers are exposed to much higher levels of PAH than the average individual (8) and yet the percentage of antibody responders did not increase. However, the amounts of BPDE-DNA specific antibodies in those sera appeared to be substantially higher as measured by both the antibody titers and the absorbance levels obtained using the ELISA. Evaluation of antibody titers to BPDE-DNA in the sera used for the current study demonstrated that most of these antibodies were present at very low levels. Only $12 \%$ of the positive samples (4/33) had antibody titers greater than 125 and a total of $58 \%$ of the positive samples (19/33) had antibody titers of only 25 . This is in contrast to the data obtained using serum samples from coke oven workers where $73 \%(8 / 11)$ of the antibody positive samples had titers of 625 or greater, whereas only $27 \%$ had the lower titers of 25 and 125 (15). These observed variations in antibody titers suggest to us that the amounts of antibody that are produced, but not the presence or absence, are correlated positively with levels of PAH exposure.

Biological variables such as human genetic differences may be involved in the control of immune responses generated against epitopes on environmental carcinogens and/or the DNA adducts. Interindividual variations have been documented with respect to human antibody responses following vaccination or infection by measles virus (25), and infection by Plasmodium falciparum (26). The ability of humans to respond to these and other antigens such as insulin from different species is under the control of the major histocompatibility system (HLA) (25-27). Similar genetic control mechanisms may be involved in the production of antibodies to epitopes on PAH-DNA adducts.

Similar genetic restraints may have been involved with the observation that antibody responses were usually broadly reactive, with antibodies being produced against multiple different forms of PAH-DNA adducts, or else totally negative. We feel it likely that only certain individuals are genetically capable of mounting an immune response to the PAH-DNA adducts but that this ability may not be specific to any particular PAH-DNA adduct. Antibody responses against many different forms of PAH-DNA adducts or a complete lack of any PAH-DNA adduct specific antibody responses would be expected if our hypothesis is valid. The genetic control of antibody responses in both the mouse and man against simple, chemically defined antigens and chemical moieties on macromolecular proteins hapten-carrier complexes, is known to be 
mediated through the genes and products of the major histocompatibility system, particularly the class II genes and antigens $(28,29)$. The chemical nature of the PAH-DNA adducts used in this study is very similar to the classical organization of chemical haptens on macromolecular carriers. We therefore believe it likely that the MHS may exert immune response gene control over the antibody responses to PAH-DNA adducts. Further experimentation using genetically defined animals or persons of known HLA types and including the measurement of other immune parameters will be required to address this issue.

Differences between the ability of individuals to metabolize PAH to form adducts with DNA and to remove or repair these adducts are known to exist $(30,31)$. Levels of metabolic oxidation of other xenobiotics have been shown to vary and to be partially under genetic control $(32,33)$. Furthermore, epidemiological data suggest that the higher rates of metabolic oxidation of at least one xenobiotic, debrisoquine, are associated with susceptibility to lung cancer (34). The ability or lack of ability of a person to metabolize PAH to form PAHDNA adducts and to produce antibodies against these PAHDNA adducts may be associated. We are currently evaluating serum samples from individuals for the presence of antibodies to PAH-DNA adducts and concurrently determining oxidation phenotypes using debrisoquine. If such a correlation does exist then metabolic oxidation phenotypes and the presence of antibodies to PAH-DNA adducts could be used either together or separately as markers to assess individual cancer risk.

We did not identify any simple significant associations between smoking status and the presence of antibodies to PAHDNA adducts. However, we did find that the production of antibodies to PAH-DNA adducts was different in males and females. The failure to observe definitive correlations in our study may not be so unexpected since exposure to PAH is not limited to the use of tobacco products. Additionally, tobacco usage may be partially immunosuppressive so that high antibody production will be limited by active cigarette smoking. The relationship between antibody production and cigarette smoking will need to be evaluated using persons with well documented smoking histories and including information about the duration of smoking cessation for past smokers. Further evaluation of epidemiological data involving the dietary habits of volunteers may also allow for a more detailed assessment of these types of data. In depth studies to address these points are in progress or are being organized.

The time of initial antigenic stimulation which lead to the production of PAH-DNA adduct specific antibodies could not be determined in this study. When one considers the potential longevity of immunological memory that can be recalled upon reexposure to antigen then it seems plausible that the initial immune stimulus could have occurred many years previously. In fact, we propose that antibodies to chemical carcinogenDNA adducts are indicators of current or past exposure to certain specific carcinogens and that these antibodies may be useful as biological tools in epidemiological studies particularly as they relate to cancer. Studies to evaluate the occurrence of antibodies to PAH-DNA adducts in cancer patients are currently in progress.

\section{Acknowledgments}

Supported in part by grants Y01-CP-30500 and N01-CP-51029 from the National Cancer Institute.

\section{References}

1. I. A. R. C. Monographs on the Evaluation of the Carcinogenic Risk of Chemicals to Humans, World Health Organization. 1983. World Health Organization, Lyon, France. 32:1-477.

2. Lee, M. L., M. Novotny, and K. D. Bartle. 1976. Gas chromatography/mass spectrometric and nuclear magnetic resonance determination of polynuclear aromatic hydrocarbons in tobacco and marijuana smoke condensates. Anal. Chem. 48:405-416.

3. Masuda, Y., and M. Kuratsune. 1972. Comparison of the yield of polycyclic aromatic hydrocarbons in smoke from Japanese tobacco. Jpn. J. Hyg. 27:339-341.

4. Grimmer, G. 1983. Profile analysis of polycyclic aromatic hydrocarbons in air. In Handbook of PAH. A. Bjorseth, editor. Marcel Dekker, New York. 149-181.

5. Grimmer, G. 1980. Changes in PAH-profiles in different areas of a city during the year. In Polynuclear Aromatic Hydrocarbons: Chemistry and Biological Effects. A. Bjorseth and A. J. Dennis, editors. Battelle Press, Columbus, OH. 107-125.

6. Olufsen, B. 1980. Polynuclear aromatic hydrocarbons in Norwegian drinking water resources. In Polynuclear Aromatic Hydrocarbons: Chemistry and Biological Effects. A. Bjorseth and A. J. Dennis, editors. Battelle Press, Columbus, OH. 333-343.

7. National Research Council. 1982. Diet, Nutrition and Cancer. National Academy Press, Washington, D.C. 14.25-14.28.

8. Bridbord, K., J. F. Finklea, J. K. Wagoner, J. B. Moran, and P. Caplan. 1976. Human exposure to polynuclear aromatic hydrocarbons. In Polynuclear Aromatic Hydrocarbons: Chemistry, Metabolism and Carcinogenesis. R. I. Freudenthal and P. Jones, editors. Raven Press, New York. 319-324.

9. Sims, P., P. L. Grover, A. Swaisland, K. Pal, and A. Hewer. 1974. Metabolic activation of benzo[a]pyrene proceeds by a diol-epoxide. Nature (Lond.) 252:326-328.

10. Jeffrey, A. M., I. B. Weinstein, K. W. Jennette, K. Grzeskowiak, K. Nakanishi, R. G. Harvey, H. Autrup, and C. Harris. 1977. Structures of benzo[a]pyrene-nucleic acid adducts formed in human and bovine bronchial explants. Nature (Lond.) 269:348-350.

11. H. V. Gelboin. 1980. Benzo[a]pyrene metabolism, activation, and carcinogenesis: role and regulation of mixed-function oxidases and related enzymes. Physiol. Rev. 60:1107-1166.

12. Grover, P. L., A. Hewer, K. Pal, and P. Sims. 1976. The involvement of a diol-epoxide in the metabolic activation of benzo[a]pyrene in human bronchial mucosa and in mouse skin. Int. J. Cancer 18:1-6.

13. Harris, C. C., B. F. Trump, R. Grafstrom, and H. Autrup. 1982. Differences in metabolism of chemical carcinogens in cultured human epithelial tissues and cells. J. Cell. Biochem. 18:285-294.

14. Shamsuddin, A. K. M., N. T. Sinopoli, K. Hemminki, R. R. Boesch, and C. C. Harris. 1985. Detection of benzo[a]pyrene: DNA adducts in human white blood cells. Cancer Res. 45:66-68.

15. Harris, C. C., K. Vahakangas, M. J. Newman, G. E. Trivers, A. Shamsuddin, N. Sinopoli, D. L. Mann, and W. E. Wright. 1985. Detection of benzo[a]pyrene diol epoxide-DNA adducts in peripheral blood lymphocytes and antibodies to the adducts in serum from coke oven workers. Proc. Natl. Acad. Sci. USA. 82:6672-6676.

16. Perera, F. P., M. C. Poirier, S. H. Yuspa, J. Nakayama, A. Jaretzki, M. M. Curnen, D. M. Knowles, and I. B. Weinstein. 1982. A pilot project in molecular cancer epidemiology: determination of benzo[a]pyrene-DNA adducts in animal and human tissues by immunoassays. Carcinogenesis. 3:1405-1410.

17. Cooper, C. S., P. L. Grover, and P. Sims. 1983. The metabolism and activation of benzo[a]pyrene. In Progress in Drug Metabolism. J. W. Bridges and L. F. Chasseaud, editors. Vol. 7. John Wiley and Sons, Ltd., New York. 295-396.

18. Pulkrabek, P., S. Leffler, I. B. Weinstein, and D. Grunberger. 1977. Conformation of DNA modified with a dihydrodiol epoxide derivative of benzo[a]pyrene. Biochemistry. 16:3127-3132. 
19. Karcher, W., R. J. Fordham, J. J. Dubois, P. G. J. M. Glaude, and J. A. M. Ligthart. 1983. Spectral Atlas of Polycyclic Aromatic Compounds. D. Reidel Dortrect Publishers, Lancaster, England.

20. Gupta, R. C. 1985. Enhanced sensitivity of ${ }^{32} \mathrm{P}$-postlabelling analysis of aromatic carcinogen: DNA adducts. Cancer Res. 45:56565662.

21. Conney, A. H. 1982. Induction of microsomal enzymes by foreign chemicals and carcinogenesis by polycyclic aromatic hydrocarbons. G. H. A. Clowes Memorial Lecture. Cancer Res. 42:48754917.

22. Shugart, L. 1985. Quantitating exposure to chemical carcinogens. In vivo alkylation of hemoglobin by benzo[a]pyrene. Toxicology. 34:211-220.

23. Kleinbaum, D. G., L. L. Kupper, and H. Morgenstern. 1982. Epidemiologic Research. Lifetime Learning Publications, Belmont, CA.

24. Rothman, K. J. 1986. Modern Epidemiology. Little, Brown and Co., Boston, MA.

25. Haverkorn, M. J., B. Hofman, N. Masurel, and J. J. van Rood. 1975. HL-A linked genetic control of immune responses in man. In Transplantation Reviews. G. Moller, editor. Munksgaard, Copenhagen, Denmark. 120-124.

26. Osoba, D., H. M. Dick, A. Voller, T. J. Goosen, T. Goosen, C. C. Draper, and G. de The. 1979. Role of the HLA complex in the antibody response to malaria under natural conditions. Immunogenetics. 8:323-338.
27. Mann, D. L., N. Mendell, C. R. Kahn, A. H. Johnson, and A. Rosenthal. 1983. In vitro lymphocyte proliferation response to therapeutic insulin components. Evidence for genetic control by the human major histocompatibility complex. J. Clin. Invest. 72:1130-1138.

28. Schwartz, R. L. 1986. Immune response genes of the murine major histocompatibility complex. Adv. Immunol. 38:31-201.

29. Giles, R. E., and J. D. Capra. 1985. Structure, function and genetics of human class II molecules. Adv. Immunol. 27:1-73.

30. Harris, C. C., H. Autrup, R. Conner, L. A. Barrett, E. M. McDowell, and B. F. Trump. 1976. Interindividual variations in binding of benzo[a]pyrene to DNA in cultured human bronchi. Science (Wash. DC). 194:1067-1069.

31. Harris, C. C. 1983. Role of carcinogens, cocarcinogens and host factors in human cancer risk. In Human Carcinogenesis. C. C. Harris and H. Autrup, editors. Academic Press, Inc., Orlando, FL. 941-970.

32. Evans, D. A. P., A. Mahgoub, T. P. Sloan, J. R. Idle, and R. L. Smith. 1980. A family and population study of the genetic polymorphism of debrisoquine oxidation in a white British population. J. Med. Genet. 17:102-105.

33. Evans, D. A. P., D. Harmer, D. Y. Downham, E. J. Whibley, J. R. Idle, J. Ritchie, and R. L. Smith. 1983. The genetic control of sparteine and debrisoquine metabolism in man with new methods of analysing bimodal distributions. J. Med. Genet. 20:321-329.

34. Ayesh, R., J. R. Idle, J. C. Ritchie, M. J. Crothers, and M. R. Hetzel. 1984. Metabolic oxidation phenotypes as markers for susceptibility to lung cancer. Nature (Lond.). 312:169-170. 